\title{
E-cigarettes, heat-not-burn and smokeless tobacco products
}

Nicotine-containing products, such as e-cigarettes and heat-not-burn products, are becoming increasingly popular as an alternative to cigarettes.

This factsheet looks at the different products available and considers whether using these products is safe for the lungs.

\section{E-cigarettes}

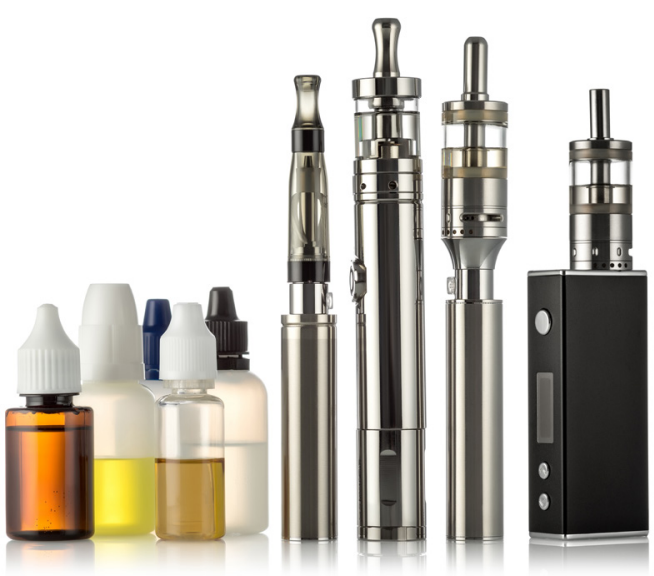

An e-cigarette is a battery-operated device that allows the user to breathe in nicotine through a vapour. They are sometimes referred to as Electronic Nicotine Delivery Systems (ENDS) and this term also includes devices such as vapes, e-hookahs, e-pipes and e-cigars. These devices work by heating a liquid so that it turns into a vapour for the user to inhale, or 'vape'. Although the vapour may look like steam, it does not contain any water. The liquid is made from chemicals called propylene glycol and/or glycerine, and it can have different flavours and different amounts of nicotine, which the user can select. Unlike traditional cigarettes, the devices do not contain tobacco.

\section{How safe are e-cigarettes?}

Research into the safety of e-cigarettes is complicated as there are differences between devices and the way people use the devices. Also e-cigarettes are constantly changing with new devices emerging; there are currently hundreds of brands and thousands of flavours available. As the products are relatively new, there are no long-term studies yet looking at the impact of e-cigarettes across someone's lifetime. Diseases caused by smoking can take 30-50 years to develop and e-cigarettes have only been in widespread use since the early 2000 s. This means that their long-term safety is not yet known. 
There are many studies that have looked into the short-term impact of e-cigarettes on health. These studies have found toxic and cancer-causing chemicals in the vapour of e-cigarettes. These chemicals were mostly, but not always, found in lower amounts in e-cigarettes compared to normal cigarettes. There is evidence that cells in the body that are exposed to vapour become damaged and stop working, and evidence showing a link between the vapour and chance of inflammation and infections in the lungs. Inflammation in the lungs can cause difficulty breathing, shortness of breath and a dry cough.

Studies have shown that healthy e-cigarette users have irritation in the airways and signs of bronchitis. Large surveys have revealed that e-cigarette users more frequently report lung symptoms than people who have never used them.

An illness known as vaping-related lung injury began to be seen in 2019. Doctors in the USA reported cases of people with symptoms including shortness of breath, fever, cough, vomiting, diarrhoea, headache, dizziness and chest pain. In some cases, these patients became very ill and were hospitalised and in the most extreme cases, a small number of patients died. The cause of this illness is not yet understood, but research is ongoing to learn more about it.

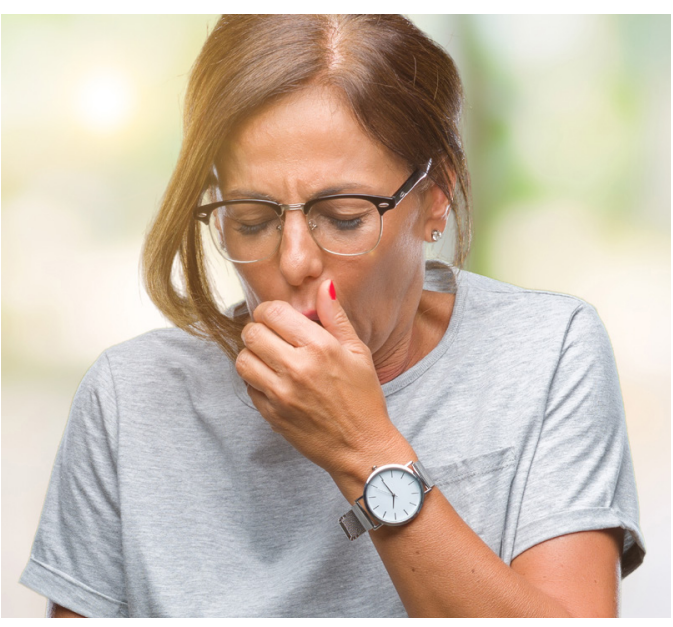

Almost all independent research has revealed some negative health effects of e-cigarettes, and existing evidence indicates that this damage will have lasting effects and lead to the development of diseases.

\section{Can e-cigarettes cause harm to others?}

It is not clear whether the vapour from an e-cigarette user can have a harmful effect on other people close by. This is known as second-hand exposure. While it is highly likely that the harm caused by second-hand exposure is less when compared with second-hand exposure to traditional cigarettes, the limited research available suggests there may be some risk, especially to vulnerable people such as the elderly, people with lung disease or pregnant women. In addition to risk of exposure, there is also a risk of harm from devices exploding or spontaneously catching fire.

Most experts believe there is harm linked to the use of e-cigarettes, but as no longterm data exist on the development of disease across a lifetime, it is not possible to conclude how harmful e-cigarettes are at this stage. 


\section{Can e-cigarettes help smokers to quit?}

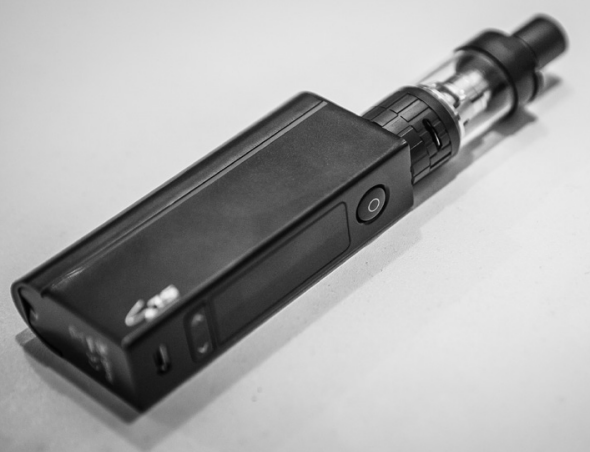

E-cigarettes are often used by smokers who are trying to quit. Usually e-cigarettes are purchased as a consumer product. A few countries are considering whether to offer e-cigarettes as part of a treatment plan to quit smoking, in a similar way to traditional quitting methods. Over 40 countries have banned the sale of e-cigarettes.

Traditional quitting methods, such as nicotine patches and gum, are usually offered by a pharmacy where a pharmacist can provide advice. The medical quitting methods are also regulated as health products with controlled levels of nicotine and the user may also be referred to psychological services for support fighting their addiction. E-cigarettes can be purchased in shops on the high street and users will not usually access any wider therapies to help them to quit. The levels of nicotine vary considerably and are not regulated by health agencies.

No e-cigarette brand has been approved as an aid to quit smoking. There are some studies that have shown that e-cigarettes can be used as a quitting aid, but there is a lack of evidence over whether these work as well as established methods. Research suggests that most adults buy e-cigarettes to quit smoking. But most of them end up using both e-cigarettes and tobacco.

There is growing evidence that using e-cigarettes and conventional cigarettes is more harmful than smoking alone. Overall, there is not enough evidence to show whether e-cigarettes can be used as a successful tool to help smokers quit.

There is evidence that the use of e-cigarettes has led to children and young adults taking up smoking, as e-cigarette users are more likely to try tobacco products. Recent surveys in the USA and some European countries have shown increases in e-cigarette use in young people. Between 2011 and 2018 in the USA, use of e-cigarettes rose from $1.5 \%$ to $20.8 \%$.

\section{Heat-not-burn tobacco products}

'Heat not burn' or 'smokeless tobacco' products are electronic devices that, unlike e-cigarettes, contain tobacco. The tobacco is heated to a high temperature, without setting it alight and creating 'smoke' that the user sucks in. They contain nicotine, additives and are often flavoured. 
There is currently no evidence showing that these products are less harmful than traditional cigarettes. In fact, more than 20 harmful chemicals were found in heatnot-burn products in higher amounts than in traditional cigarette smoke; exposure to harmful chemicals is also higher compared to e-cigarettes. There is some evidence that

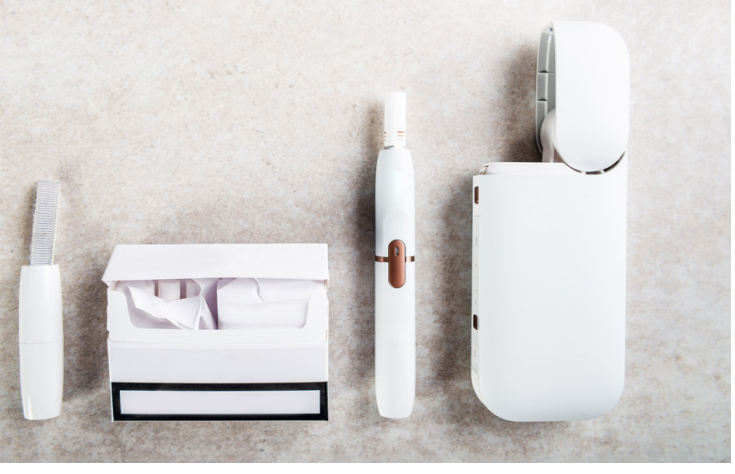
new chemicals exist in heat-not-burn products that are not present in traditional cigarettes, and which could be toxic and harmful. Studies in both animals and humans have suggested the products cause harm to the lungs. Studies have also shown no improvement in lung function or inflammation in the lungs in smokers who switched to heated tobacco.

The products are also highly variable between different brands, but there is some evidence that the toxins found in the vapour are capable of causing cancer. There is no evidence to support claims that smokers switch from traditional cigarettes to exclusive use of heat-not-burn tobacco products. There is currently insufficient evidence on whether second-hand exposure is harmful.

\section{Smokeless tobacco}

Smokeless tobacco can be chewed or placed on the inside of your lip (this type is called snus). It releases tobacco and nicotine, which gets into the bloodstream through the gums. Users often spit a lot during use as the process produces a lot of saliva.

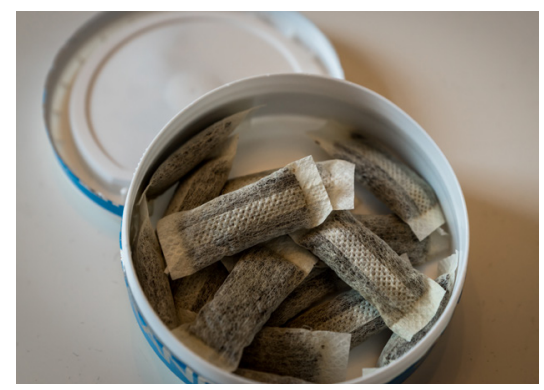

The composition of smokeless tobacco products varies, but many have been found to include cancer-causing chemicals. Research has linked the use of smokeless tobacco to cancer and other diseases of the mouth, and with heart disease and stroke. Evidence of the risk of cancer due to the use of snus is inconclusive.

Tobacco is toxic and contains toxins that are capable of causing cancer in all forms. The World Health Organization considers that all forms of tobacco use are harmful, including e-cigarettes, heat-not-burn products and smokeless tobacco. 


\section{Tobacco harm reduction strategies}

This factsheet has given a summary of the evidence available on the safety of products that claim to reduce the amount of harm for users compared with traditional cigarettes.

'Tobacco harm reduction' is a phrase that describes an overall approach to reduce the harm caused by cigarette smoking. There is some debate among policymakers whether this strategy should be implemented to help people stop smoking. Some experts believe that reducing the amount of toxins in the body is the most important thing, even if some still remain. Others believe that we should not be recommending the use of products that have any damaging effects, even if they are reduced.

As organisations working in lung health, the advice from the European Respiratory Society (ERS) and European Lung Foundation (ELF), is as follows:

"The human lungs are created to breathe clean air, not toxins and carcinogens, and the human body is not meant to be dependent on addictive drugs. ERS and ELF cannot recommend any product that is damaging to the lungs and human health."

ELF Chair, Isabel Saraiva, does not advocate the use of e-cigarettes and believes the lungs are made for clean air only. She comments: "As an ex-smoker, I understand that it is tempting for people, who think that it will be easier to stop smoking by substituting cigarettes with e-cigarettes. If they had been available when I was

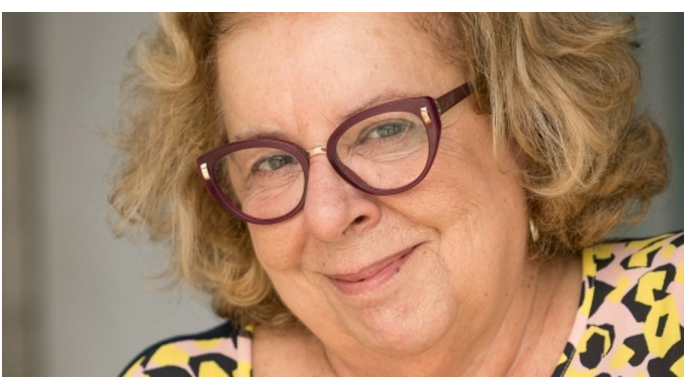
trying to stop, I would not have been able to break my addiction to nicotine. Lungs are made for clean air and clean air only. I believe e-cigarettes are dangerous, particularly for children. It is my view that the only possible way to quit smoking for good is with professional help from healthcare workers."

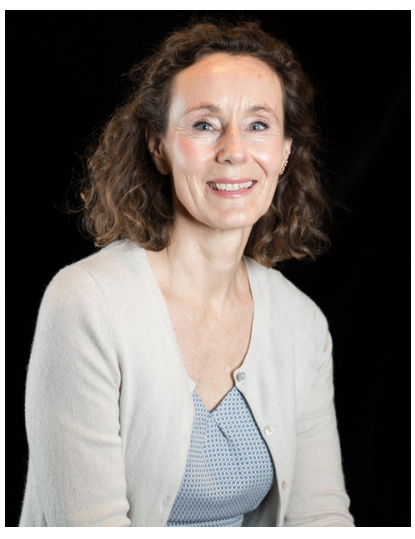

Charlotta Pisinger is Chair of the ERS Tobacco Control Committee and Clinical Professor of Tobacco Control at Bispebjerg and Frederiksberg Hospital and the University of Copenhagen, Denmark. Professor Pisinger explains the ERS and ELF position: "Tobacco harm reduction strategies are based on incorrect assumptions that smokers cannot or will not quit smoking, but in reality, the majority of smokers want to quit and they dislike being nicotine dependent. Most of the new products, including heated tobacco and e-cigarettes, 
are devices of nicotine inhalation, and therefore do not help smokers to beat their addiction to nicotine. Furthermore, the harm reduction strategy does not take into account that most users do not switch completely from cigarettes but continue smoking and vaping. Also, an increasing number of non-smokers use these products, and in adolescents they seem to be a first step towards users smoking traditional cigarettes."

\section{Further information}

\section{European Lung Foundation | www.europeanlung.org}

The European Lung Foundation (ELF) has a range of factsheets (available in up to 29 languages) on lung conditions and lung health, which are available to download from the ELF website.

\section{European Respiratory Society | www.ersnet.org | www.ersjournals.com} The European Respiratory Society (ERS) publishes information and original research for healthcare professionals and scientists in its journals and other academic resources.

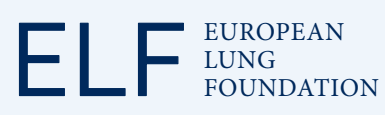

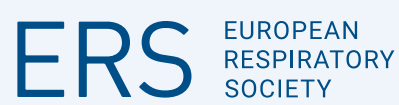

The European Lung Foundation (ELF) was founded by the European Respiratory Society (ERS) in 2000 with the aim of bringing together patients, the public and respiratory professionals to positively influence lung health.

This factsheet was produced in March 2020. 